\title{
A SPECIAL CALABI-YAU DEGENERATION WITH TRIVIAL MONODROMY
}

\author{
SLAWOMIR CYNK AND DUCO VAN STRATEN
}

\begin{abstract}
A well-known theorem of Kulikov, Persson and Pinkham states that a degeneration of a family of K3-surfaces with trivial monodromy can be completed to a smooth family. We give a simple example that an analogous statement does not hold for Calabi-Yau threefolds.
\end{abstract}

\section{INTRODUCTION}

In the study of degenerations of $K 3$ surfaces, the theorems of Kulikov [11 Persson and Pinkham [15] are fundamental and play a key role in the proof of the surjectivity of the period map for $K 3$ surfaces. One important result concerns degenerations of type $I:$ if $f: \mathcal{X} \longrightarrow \Delta$ is a degeneration of $K 3$ surfaces over the disc $\Delta:=\{t \in \mathbb{C}|| t \mid<1\}$, with monodromy on $H^{2}\left(X_{t}\right)$ of finite order, then after an appropriate base change and birational modification of the zero-fibre we obtain a family for which the fibre $X_{0}$ over 0 is smooth. For example, if $\mathcal{X} \longrightarrow \Delta$ acquires only ADE-singularities at the zero-fibre, then the theorem is implied by to the phenomenon of simultaneous resolution after base change for these singularities, discovered by Brieskorn [2], generalising the $A_{1}$-case of Atiyah [1]. The fact that for ADE-singularities in dimension two resolving and deforming 'are the same' has been recognized as a typical feature in hyperkähler geometry and recently a generalisation of the above theorem to degenerations of higher dimensional hyperkähler manifolds was given in [10]. As remarked in that paper, it is known that this does not generalise to degenerations of higher dimensional Calabi-Yau varieties. The homological monodromy of an odd dimensional $A_{1}$-singularity has infinite order, but the $A_{2}$-singularity has finite order. It was remarked long ago by Clemens, Friedman [9] and Morgan [14 that a Calabi-Yau 3-fold degeneration acquiring an $A_{2}$-singularity does not have a smooth filling and there is a result of Voisin [21] implying a same result for four dimensional varieties aquiring an $A_{1}$-singularity.

The purpose of this paper is to present a completely different type of example and analyse it in some detail. The main result is the following

\section{Theorem}

There exists a flat family $f: \mathcal{Y} \longrightarrow \Delta$ of projective threefolds such that

(1) $\mathcal{Y}$ is a smooth fourfold,

(2) for $t \neq 0$, the fibre $Y_{t}:=f^{-1}(t)$ is a smooth Calabi-Yau threefold with

$$
h^{1,1}\left(Y_{t}\right)=41, \quad h^{1,2}\left(Y_{t}\right)=1,
$$

(3) the singular locus of $Y_{0}$ is a line $L, Y_{0}$ is double along $L$ with exactly four pinch points, 
(4) the elliptic curve $E$ doubly covering $L$ and ramified over the set $\Sigma$ of these four pinch points has $j$-invariant equal to 1728 ,

(5) the blow-up of $Y_{0}$ in $L$ is a smooth Calabi-Yau threefold $Z_{0}$ with

$$
h^{1,1}=46, \quad h^{1,2}=0,
$$

(6) the local system $H^{i}\left(Y_{t}\right)$ has trivial monodromy over $\Delta^{*}$ for $i \neq 3$ and $\mathbb{Z} / 2 \mathbb{Z}$ monodromy for $i=3$.

\section{Corollary}

The semi-stable reduction, obtained after a base change $t \mapsto t^{2}$, has trivial monodromy and the special fibre consists of two components, one of which is a projective, smooth and rigid Calabi-Yau manifold and the other is a smooth quadric bundle.

In the Clemens-Friedman-Morgan example we have a Calabi-Yau variety that acquires an $A_{2}$-singularity, which is a terminal singularity. So from the point of view of the minimal model program, this singular variety should be considered as good as a smooth one. In fact, there does not exist a crepant resolution of the singular member. In our example the zero-fibre $Y_{0}$ has canonical singularities, and admits a crepant resolution to a honest smooth Calabi-Yau variety $Z_{0}$. There is a change in cohomology in going from $Z_{0}$ to $Y_{t}$. In section 4 we will see that the third limiting Hodge structure splits

$$
H_{\text {lim }}^{3}\left(Y_{\infty}, \mathbb{Q}\right)=H^{3}\left(Z_{0}, \mathbb{Q}\right) \oplus H^{1}(E, \mathbb{Q})(-1)
$$

where $E$ is the elliptic curve mentioned in the theorem. The two pieces of this decomposition are necessarily supported by two different irreducible components of the zero fibre.

The structure of the paper is as follows. In section 1 we describe the basic structure of the example. In section 2 we describe the resolution process in some detail and give the proof of the above theorem. In section 3 we analyse the example on a cohomological level. In section 4 we collect some remarks and speculations.

\section{The eXAmple}

2.1. Double octics. Our example is based on certain special double octics, double covers of $\mathbb{P}^{3}$ ramified along an arrangement of eight planes $P_{1}, P_{2}, \ldots, P_{8}$. Such a space can be given as a hypersurface in a weighted projective space

$$
\left\{u^{2}=L_{1} L_{2} L_{3} L_{4} L_{5} L_{6} L_{7} L_{8}\right\} \subset \mathbb{P}[4,1,1,1,1],
$$

where $L_{i}$ is a linear form defining the plane $P_{i}$. For a generic choice of the planes $P_{i}$, the branch divisor $D=\cup_{i=1}^{8} P_{i}$ (and hence also the double cover) has 28 double lines and 56 triple points, along which it is singular. By blowing up the (strict transforms of) the double lines in any order we obtain a crepant resolution which is a Calabi - Yau threefold with Hodge numbers $h^{12}=9, h^{11}=29$. For special positions of the planes the singularities of the double octic change, but as long as the configuration of planes does not have 4-fold lines or 6-fold points, there still exists a crepant resolution, but now the Hodge numbers can take various values, depending on the precise properties of the configuration. Recently, all different cases have been listed. For more information on special double octics we refer to [13], [5]. 
Our example is based on the following family of double octics

$$
X_{t}:=\left\{u^{2}=x y(x+y) z(x+2 y+z+t v) v(y+z+v)(x+y+z+(t-1) v)\right\} .
$$

where $t \in \mathbb{P}^{1}$ is considered as the parameter. We label the linear forms $L_{1}, L_{2}, \ldots, L_{8}$ in the order as they are written in the above equation:

$$
L_{1}=x, \quad L_{2}=y, \quad L_{3}=x+y, \ldots, \quad L_{8}=x+y+z+(t-1) v .
$$

For $t \neq 0,1,2, \infty$ this configuration has exactly

- a single triple line $\ell_{\text {triple }}: x=y=0$,

- 25 double lines,

- six fourfold points,

$(1: 0: 0: 0), \quad(0: 1:-1: 0),(1:-1: 1: 0), \quad(t-2: 1: 0:-1), \quad(1: 0:-1: 0), \quad(1:-1: 0: 0)$ not lying on the triple line $\ell_{\text {triple }}$ (called points of type $p_{4}^{0}$ ),

- five fourfold points

$$
(0: 0: 1: 0),(0: 0: 0: 1), \quad(0: 0:-t: 1), \quad(0: 0: 1:-1), \quad(0: 0: t-1:-1)
$$

on the triple line $\ell_{\text {triple }}$ (called points of type $p_{4}^{1}$ ).

This arrangement is projectively equivalent to arrangement No. 153 in [13] via the coordinate transformation

$$
(x, y, z, v) \mapsto(-y-z-v, v, y, x+y+z+(t+1) v),
$$

and reparametrisation $t \mapsto t-2$.

We are here concerned specifically with the degeneration that occurs at $t=0$ and consider the family of double octics over the unit disc:

$$
\pi: \mathcal{X} \rightarrow \Delta
$$

with fibre over $t \in \Delta$ the double octic $X_{t}$ defined by the above equation. For this degeneration, there are two important lines, namely the triple line $\ell_{\text {triple }}$ and the moving line $m_{t}=P_{4} \cap P_{5}$. If $t$ goes to 0 , the moving line intersects the triple line, and the two 4-fold points $\ell_{\text {triple }} \cap P_{4}$ and $\ell_{\text {triple }} \cap P_{5}$ move together to form a 5 -fold point. The result is that for $t=0$ we obtain a configuration equivalent to the rigid arrangement No. 93 of 13 .

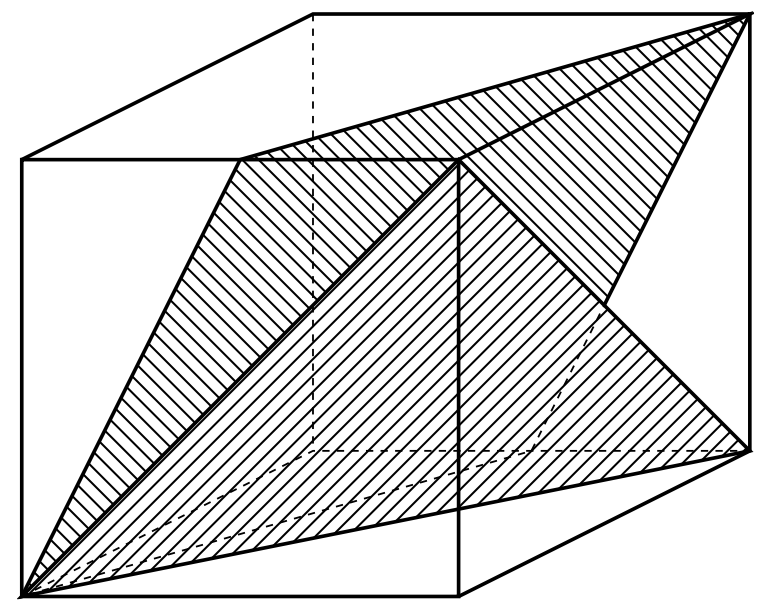

The plane spanned by the lines $\ell_{\text {triple }}$ and $m_{0}$ makes a fourth plane $P$ through $\ell_{\text {triple }}$. This plane does not belong to the octic arrangement, but the the planes $P_{1}, P_{2}, P_{3}, P$ define four points on the projective line $L$ of all planes through $\ell_{\text {triple }}$. We let $E$ be 
the double cover of $L$ ramified over these four points. As these planes are in harmonic position, the $j$-invariant of $E$ is seen to be 1728 . This is the elliptic curve we were alluding to in the introduction. We note furthermore that all strata of the singular locus of $X_{t}$ are defined by the intersections among the planes $P_{i}$. As these are not interchanged by the monodromy, these loci form trivial families over $\Delta^{*}$.

2.2. Construction of $\mathcal{Y} \longrightarrow \Delta$. We will now construct the family $f: \mathcal{Y} \longrightarrow \Delta$ of the theorem from the family of singular double octics $\mathcal{X} \longrightarrow \Delta$ by a certain specific sequence of blow-ups. To be more precise, we construct a sequence of blow-ups and a diagram of two-fold covers over it:

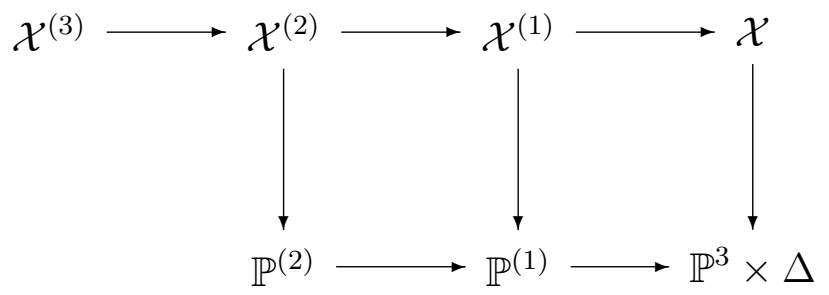

We are dealing here with families over $\Delta$ and by blowing-up a line we mean the blowingup a relative line over $\Delta$, which is really a surface.

- Blow-up in $\mathbb{P}^{3} \times \Delta$ first in the locus of all fourfold points of type $p_{4}^{0}$ and the triple line. Call the resulting space $\mathbb{P}^{(1)}$ and let $D^{(1)}$ be the strict transform of $D=D^{(0)}$, plus the divisor $E:=L \times \ell_{\text {triple }} \times \Delta=\mathbb{P}^{1} \times \mathbb{P}^{1} \times \Delta$ lying over the triple line $\ell_{\text {triple }} \times \Delta$ over $\Delta$. We denote by $\mathcal{X}^{(1)}$ the double cover ramified over $D^{(1)}$. The branch divisor $D^{(1)}$ contains, apart from the strict transforms of the 25 double lines of $D$ also eight 'new' double lines: three lines $m_{1}, m_{2}, m_{3}$ in one ruling corresponding to the three planes $P_{1}, P_{2}, P_{3}$ containing the triple line $\ell_{\text {triple }}$, and five further lines $m_{4}, \ldots, m_{8}$ in the second ruling, namely the intersection of the strict transforms of the planes $P_{4}, P_{5}, \ldots, P_{8}$. In local coordinates near the line $m_{1}$ the space $\mathcal{X}^{(1)}$ is described by the equation

$$
u^{2}=x y z(x+2 x y+z+t) F .
$$

Here the divisor $x=0$ is the exceptional set $E, y=0$ defines the line $m_{1}$. The next two factors $z=0$ and $x+2 x y+z+t=0$ are the equations of the strict transforms of $L_{4}$ resp $L_{5}$. The factor $F$ is the product of all other factors; it is non-zero on $m_{1}$. (We shall consider only the resolution of the complement of lines $m_{2}$ and $m_{3}$, as the resolution in neighbourhoods of these two lines is completely analogous to what happens near $m_{1}$ ).

- In the next step we blow-up all double curves, except three of them: $m_{4}, m_{5}$ and the intersection of strict transforms of planes $P_{4}$ and $P_{5}$. The new branch divisor $D^{(2)}$ is the strict transform of $D^{(1)}$. Call the resulting double cover $\mathcal{X}^{(2)}$. Over the blow - up of the line $m_{1}$ the space $\mathcal{X}^{(2)}$ is described in two charts by

$$
\begin{aligned}
& (x, y) \longmapsto(x y, y), \quad u^{2}=x z\left(x y+2 x y^{2}+z+t\right) F . \\
& (x, y) \longmapsto(x, x y), \quad u^{2}=y z\left(x+2 x^{2} y+z+t\right) F
\end{aligned}
$$

- The last step of the resolution is to blow-up the double cover in the union of singular loci of all fibers $\bigcup_{t \in \Delta} \operatorname{Sing}\left(X_{t}\right)$. We denote by $\mathcal{Y}=\mathcal{X}^{(3)}$ the resulting variety. To analyse what we have, we look in the two charts of $\mathcal{X}^{(2)}$ around the blow-up of $m_{1}$. In the second chart the branch-divisor and the fibre of the 
branch-divisor are both simple normal crossings. As a consequence, the parts of the spaces $\mathcal{Y}$ and $Y_{t}$ lying over this chart are smooth. To analyse $\mathcal{Y}$ in the first chart, we have to blow-up $\mathcal{X}^{(2)}$ in the ideal

$$
\left(x z, x\left(x y+2 x y^{2}+z+t\right), z\left(x y+2 x y^{2}+z+t\right), u\right) .
$$

This blow-up is given as the closure of the map

$$
(x, y, z, t, u) \mapsto(X, Y, Z, T)=\left(x z, x\left(x y+2 x y^{2}+z+t\right), z\left(x y+2 x y^{2}+z+t\right), u\right)
$$

Using Singular [6] we verified that $\mathcal{Y}$ is smooth in this chart as well and the special fibre is singular along the line

$$
\operatorname{Sing}\left(Y_{0}\right)=(x=z=u=X=Y=Z=0)
$$

is contained in the affine chart $T=1$, moreover in this affine chart the variety $Z$ is locally given as

$$
x=X Y, z=X Z, u=X Y Z,\left(y+2 y^{2}\right) X Y+X Z-Y Z+t=0 .
$$

\section{proof of the Theorem:}

Hodge numbers: The statement about the Hodge numbers follow from the formulas that express the Hodge-numbers of a resolved double octics $X_{t}$ and are recorded in [13]: $h^{1,1}=46, h^{1,2}=0$ for $X_{0}$ and $h^{1,1}=41, h^{1,2}=1$ for $X_{t}, t \in \Delta^{*}$. As the Hodge numbers do not depend on the choice of the resolution, we find the Hodge numbers as stated in the theorem.

Properties of $Y_{0}$ : The fact that $Y_{0}$ is double along the line $L$ and is resolved by a single blow-up follows from the above local calculations. The singular line $L$ of $Y_{0}$ can be identified with the pencil of planes through the triple line $\ell_{\text {triple }}$ at the fivefold point $(0: 0: 0: 1)$. Three of the pinch-points correspond to three planes containing $\ell_{\text {triple }}$, the fourth pinch-point is the direction of the intersection line $P_{4} \cap P_{5}$.

Monodromy: The cohomology group $H^{2}\left(Y_{t}, \mathbb{C}\right), t \in \Delta^{*}$ is generated by classes of components of the exceptional locus of the crepant resolution, so it has trivial monodromy. A simple way to determine the monodromy on $H^{3}\left(Y_{t}\right)$ is using the Picard-Fuchs operator $\mathcal{P}$ of the family that was determined in [4.

$$
\begin{aligned}
& \mathcal{P}=4 \Theta\left(\Theta-\frac{1}{2}\right)\left(\Theta-\frac{3}{2}\right)(\Theta-2)-12 t\left(\Theta-\frac{1}{2}\right)^{2}\left(\Theta^{2}-\Theta+\frac{1}{12}\right)+13 t^{2}\left(\Theta^{4}+\frac{9}{26} \Theta^{2}+\frac{1}{208}\right) \\
& -6 t^{3}\left(\Theta+\frac{1}{2}\right)^{2}\left(\Theta^{2}+\Theta+\frac{7}{13}\right)+t^{4}\left(\Theta+\frac{1}{2}\right)(\Theta+1)^{2}\left(\Theta+\frac{3}{2}\right) \in \mathbb{Q}\langle t, \Theta\rangle,
\end{aligned}
$$

where $\Theta=t \partial / \partial t$ is the logarithmic derivative with respect to the parameter $t$. The Riemann symbol, that collects the exponents at all singular points is:

$$
\left\{\begin{array}{cccc}
2 & 1 & 0 & \infty \\
\hline 0 & 0 & 0 & 1 / 2 \\
1 / 2 & 1 / 2 & 1 / 2 & 1 \\
1 / 2 & 1 / 2 & 3 / 2 & 1 \\
1 & 1 & 2 & 3 / 2
\end{array}\right\}
$$

The local system $\mathcal{S}$ ol of solutions on $\mathbb{P}^{1} \backslash\{0,1,2, \infty\}$ is isomorphic to the local system with fibres $H^{3}\left(Y_{t}, \mathbb{C}\right)$. It can be checked by computing the formal solutions around 0 that no logarithms occur in the solutions and one finds four series solutions

$$
\begin{gathered}
\phi_{0}(t)=1+\frac{1}{4} t-\frac{23}{1120} t^{2}+\ldots, \quad \phi_{1}(t)=t^{1 / 2}\left(1+t+\frac{4}{5} t^{2} \ldots\right), \\
\phi_{2}(t)=t^{3 / 2}\left(1+t+\frac{6}{7} t^{2}+\ldots\right), \quad \phi_{3}(t)=t^{2}\left(1+\frac{5}{4} t+\ldots\right)
\end{gathered}
$$


Consequently monodromy for $H^{3}\left(Y_{t}\right), t \in \Delta^{*}$ has order 2 .

\section{Cohomological analysis}

To describe the cohomological relation between the singular fibre $Y_{0}$ and the generic fibre $Y_{t}$ of our family $f: \mathcal{Y} \rightarrow \Delta$, we use the nearby and vanishing cycle formalism from [18]. There is a distinguished triangle in $D_{\text {constr }}^{b}\left(Y_{0}, \mathbb{Q}\right)$ that reads

$$
\ldots \longrightarrow \mathbb{Q}_{Y_{0}} \longrightarrow R \Psi_{f}(\mathbb{Q}) \longrightarrow R \Phi_{f}(\mathbb{Q}) \stackrel{+1}{\longrightarrow} \ldots
$$

and which leads to a long exact sequence in cohomology

$$
\ldots \longrightarrow H^{k}\left(Y_{0}, \mathbb{Q}\right) \longrightarrow \mathbb{H}^{k}\left(R \Psi_{f}(\mathbb{Q})\right) \longrightarrow \mathbb{H}^{k}\left(R \Phi_{f}(\mathbb{Q})\right) \longrightarrow \ldots
$$

The cohomology group $H^{k}\left(Y_{0}, \mathbb{Q}\right)$ carries, after Deligne [7, a natural mixed Hodge structure. The hypercohomology $\mathbb{H}^{k}\left(R \Psi_{f}(\mathbb{Q})\right)$ can be identified with the cohomology $H^{k}\left(Y_{\infty}, \mathbb{Q}\right)$ of the nearby fibre and carries a canonical mixed Hodge structure after Schmid [19] and Steenbrink [20]. The vanishing cohomology groups

$$
\mathbb{H}^{k}:=\mathbb{H}^{k}\left(R \Phi_{f}(\mathbb{Q})\right)
$$

can be given a mixed Hodge structure in a way compatible with this exact sequence.

Proposition: One has

$$
\mathbb{H}^{k}=0 \text { for } k \neq 3
$$

and there is a short exact sequence

$$
0 \longrightarrow H^{1}\left(R^{2} \Phi_{f}(\mathbb{Q})\right) \longrightarrow \mathbb{H}^{3} \longrightarrow H^{0}\left(R^{3} \Phi_{f}(\mathbb{Q})\right) \longrightarrow 0
$$

and identifications

$$
H^{1}\left(R^{2} \Phi_{f}(\mathbb{Q})\right)=H^{1}(E)(-1), \quad H^{0}\left(R^{3} \Phi_{f}(\mathbb{Q})\right)=\oplus_{p \in P} \mathbb{Q}(-2) \cdot[p]
$$

as MHS.

proof: We use the hypercohomology spectral sequence $\mathbb{H}^{p}\left(R \Phi_{f}^{q}(\mathbb{Q})\right) \Longrightarrow \mathbb{H}^{p+q}$. As the singular locus is $L$, which is codimension 2 in $Y_{0}$, we have that $R^{0} \Phi_{f}(\mathbb{Q})=R^{1} \Phi_{f}(\mathbb{Q})=$ 0 . At a general point $q \in L$ the threefold $Y_{0}$ has a transverse $A_{1}$-singularity, hence we have that the stalk $R^{2} \Phi_{f}(\mathbb{Q})_{q}$ is one-dimensional, whereas $R^{3} \Phi_{f}(\mathbb{Q})_{q}=0$. At the pinchpoints $p \in \Sigma$ one has $R^{2} \Phi_{f}(\mathbb{Q})_{p}=0$ and $R^{3} \Phi_{f}(\mathbb{Q})_{p}$ one-dimensional. So $R^{3} \Phi_{f}(\mathbb{Q})$ is a sky-scraper sheaf at the pinch points $\Sigma$, whereas $R^{2} \Phi_{f}(\mathbb{Q})$ is a rank one-local system on $L \backslash \Sigma$, extended to zero. From the local normal form of the pinch-point, one sees that the monodromy on $R^{2} \Phi_{f}(\mathbb{Q})$ around the points $p \in \Sigma$ are multiplication by $(-1)$. Hence there is an exact sequence

$$
0 \longrightarrow \mathbb{Q}_{L} \longrightarrow n_{*}\left(\mathbb{Q}_{E}\right) \longrightarrow R^{2} \Phi(\mathbb{Q})(1) \longrightarrow 0,
$$

where $n: E \longrightarrow L$ is the elliptic curve over $L$, ramified at $\Sigma$. From the long exact cohomology sequence we immediately obtain

$$
H^{0}\left(R^{2} \Phi_{f}(\mathbb{Q})\right)=0, \quad H^{1}\left(R^{2} \Phi_{f}(\mathbb{Q})\right)=H^{1}(E, \mathbb{Q})(-1), \quad H^{2}\left(R^{2} \Phi_{f}(\mathbb{Q})\right)=0 .
$$

The hypercohomology spectral sequences collapses and give the above result.

Corollary: The Hodge structures $H^{k}\left(Y_{0}, \mathbb{Q}\right)$ are pure of weight $k$. There are isomorphisms

$$
H^{k}\left(Y_{0}, \mathbb{Q}\right) \approx H^{k}\left(Y_{\infty}, \mathbb{Q}\right) \text { for } k \neq 3,4
$$


and short exact sequences

$$
\begin{gathered}
0 \longrightarrow H^{3}\left(Y_{0}, \mathbb{Q}\right) \longrightarrow H^{3}\left(Y_{\infty}, \mathbb{Q}\right) \longrightarrow H^{1}(E, \mathbb{Q})(-1) \longrightarrow 0 \\
0 \longrightarrow \oplus_{p \in \Sigma} \mathbb{Q}(-2) p \longrightarrow H^{4}\left(Y_{0}, \mathbb{Q}\right) \longrightarrow H^{4}\left(Y_{\infty}, \mathbb{Q}\right) \longrightarrow 0
\end{gathered}
$$

Hence we have:

$$
h^{2}\left(Y_{0}\right)=h^{2}\left(Y_{\infty}\right)=41, \quad h^{3}\left(Y_{0}\right)=2, \quad h^{4}\left(Y_{0}\right)=h^{4}\left(Y_{\infty}\right)+4=45 .
$$

proof: Note that the Hodge structures $H^{k}\left(Y_{\infty}, \mathbb{Q}\right)$ are pure, as the monodromy is trivial. As only $\mathbb{H}^{3} \neq 0$, we get from the long exact cohomology sequence isomorphisms and a five term exact sequence

$$
0 \longrightarrow H^{3}\left(Y_{0}, \mathbb{Q}\right) \longrightarrow H^{3}\left(Y_{\infty}, \mathbb{Q}\right) \longrightarrow \mathbb{H}^{3} \longrightarrow H^{4}\left(Y_{0}, \mathbb{Q}\right) \longrightarrow H^{4}\left(Y_{\infty}, \mathbb{Q}\right) \longrightarrow 0
$$

From the fact that $H^{3}\left(Y_{0}, \mathbb{Q}\right) \neq H^{3}\left(Y_{\infty}, \mathbb{Q}\right)$ and the fact that $H^{3}\left(Y_{\infty}, \mathbb{Q}\right)$ is pure, we see the only possibility is that $H^{3}\left(Y_{\infty}, \mathbb{Q}\right)$ surjects on the weight 3 part $H^{1}(E, \mathbb{Q})(-1)$ of $\mathbb{H}^{3}$ and then the kernel of $H^{4}\left(Y_{0}, \mathbb{Q}\right) \longrightarrow H^{4}\left(Y_{\infty}, \mathbb{Q}\right)$ is equal to the weight 4 quotient of $\mathbb{H}^{3}$.

3.1. Cohomology of $Z_{0}$. The space $Z_{0}$ is obtained from $Y_{0}$ by a single blow-up in the line $L$. The preimage of $L$ is a conic-bundle $Q \longrightarrow L$. So we get a diagram

$$
\begin{array}{llll}
Q & \hookrightarrow & Z_{0} \\
\downarrow & & \downarrow \pi \\
L & \hookrightarrow & Y_{0}
\end{array}
$$

The following calculation provides an independent determination of the cohomology of $Z_{0}$.

Proposition: There are exact sequences of mixed Hodge structures

$$
\begin{aligned}
0 \longrightarrow H^{2}\left(Y_{0}, \mathbb{Q}\right) \longrightarrow H^{2}\left(Z_{0}, \mathbb{Q}\right) \longrightarrow H^{0}\left(R^{2} \pi_{*} \mathbb{Q}_{z_{0}}\right) \longrightarrow 0 . \\
H^{3}\left(Y_{0}, \mathbb{Q}\right) \approx H^{3}\left(Z_{0}, \mathbb{Q}\right), \\
0 \longrightarrow H^{4}\left(Y_{0}, \mathbb{Q}\right) \longrightarrow H^{4}\left(Z_{0}, \mathbb{Q}\right) \longrightarrow H^{2}\left(R^{2} \pi_{*} \mathbb{Q}_{z_{0}}\right) \longrightarrow 0 .
\end{aligned}
$$

Furthermore:

$$
H^{0}\left(R^{2} \pi_{*} \mathbb{Q}_{Z_{0}}\right)=\mathbb{Q}(-1)^{5}, \quad H^{2}\left(R^{2} \pi_{*} \mathbb{Q}_{Z_{0}}\right)=\mathbb{Q}(-2)
$$

hence one gets

$$
h^{2}\left(Z_{0}\right)=h^{2}\left(Y_{0}\right)+5=46, \quad h^{3}\left(Z_{0}\right)=h^{3}\left(Y_{0}\right)=2, \quad h^{4}\left(Z_{0}\right)=h^{4}\left(Y_{0}\right)+1=46 .
$$

proof: In order to compare the cohomology of $Z_{0}$ and $Y_{0}$, we use the Leray spectral sequence

$$
E_{2}^{p, q}=H^{p}\left(R^{q} \pi_{*} \mathbb{Q}_{Z_{0}}\right) \Longrightarrow H^{p+q}\left(Z_{0}, \mathbb{Q}\right) .
$$

The inverse image of $L$ in $Y$ is a conic bundle $Q \longrightarrow L$. The general fibre is a smooth conic; the fibres over $p \in \Sigma$ are line pairs. Hence

$$
R^{0} \pi_{*} \mathbb{Q}_{Z_{0}}=\mathbb{Q}_{Y_{0}}, \quad R^{k} \pi_{*} \mathbb{Q}_{Z_{0}}=0, \quad k \neq 0,2
$$

From the study of the vanishing cohomology for the family $Q \longrightarrow L$ near the line-pairs, we obtain a split exact sequence

$$
0 \longrightarrow \mathbb{Q}_{\Sigma} \longrightarrow R^{2} \pi_{*} \mathbb{Q}_{Q} \longrightarrow \mathbb{Q}_{L} \longrightarrow 0
$$

which indeed leads to

$H^{0}(Q)=\mathbb{Q}, \quad H^{1}(Q, \mathbb{Q})=0, \quad H^{2}(Q, \mathbb{Q})=\mathbb{Q}(-1)^{6}, \quad H^{3}(Q, \mathbb{Q})=0, \quad H^{4}(Q, \mathbb{Q})=\mathbb{Q}(-2)$. 
The differential

$$
d_{3}: H^{0}\left(R^{2} \pi_{*} \mathbb{Q}_{Z_{0}}\right) \rightarrow H^{3}\left(R^{0} \pi_{*} \mathbb{Q}_{Z_{0}}\right)=H^{3}\left(Y_{0}, \mathbb{Q}\right)
$$

has to be zero because of weights, so we obtain from the spectral sequence short exact sequences as stated above.

3.2. The semi-stable reduction. Denote by $g: \mathcal{Z} \rightarrow \mathcal{Y}$ the blow-up of the smooth space $\mathcal{Y}$ in the line $L$. We denote the exceptional divisor of this blow-up by $P$; it is a $\mathbb{P}^{2}$-bundle over $L$. As the multiplicity of $Y_{0}$ alone $L$ is two, the divisor of the composed function

$$
h:=f \circ g: \mathcal{Z} \rightarrow \Delta
$$

is

$$
Z_{0}+2 P
$$

where the strict transform $Z_{0}$ of $Y_{0}$ is blow-up of $Y_{0}$ in $L$, hence smooth. The intersection of these two components is the surface

$$
Q:=Z_{0} \cap P .
$$

The map $Q \rightarrow L$, obtained as restriction of $g$, gives $Q$ the structure of a conic bundle over $L$; above the four pinch-points the conics degenerate into a line pair. Now we take the pull-back of $h: \mathcal{Z} \rightarrow \Delta$ by the squaring map $s: t \mapsto t^{2}$, and denote its normalisation by $\widetilde{\mathcal{Z}}$ :

$$
\widetilde{\mathcal{Z}}:=\widetilde{\times_{\Delta} \mathcal{Z}}
$$

We let $n: \widetilde{\mathcal{Z}} \rightarrow \mathcal{Z}$ the natural map, so we have the diagram

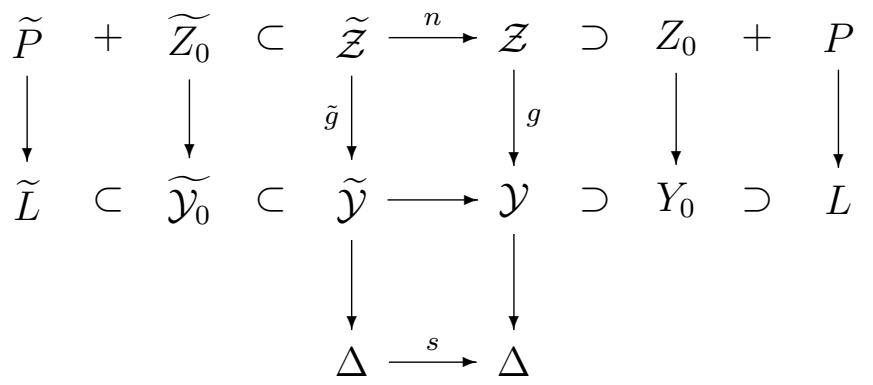

Proposition: The space $\widetilde{\mathcal{Z}}$ is smooth. The divisor

$$
\widetilde{h}^{-1}(0)=\widetilde{Z_{0}}+\widetilde{P}
$$

is reduced and normal crossing. The map $n$ induces an isomorphism

$$
\widetilde{Z_{0}} \rightarrow Z_{0}
$$

and a 2 -to-1 covering $\widetilde{P} \rightarrow P$ ramified precisely along $Q \subset P$.

The cohomology groups $H^{i}(\widetilde{P}, \mathbb{Q})$ are given by

\begin{tabular}{|c|c|c|c|c|c|c|}
\hline$H^{0}$ & $H^{1}$ & $H^{2}$ & $H^{3}$ & $H^{4}$ & $H^{5}$ & $H^{6}$ \\
\hline $\mathbb{Q}$ & 0 & $\mathbb{Q}(-1)^{2}$ & $H^{1}(E)(-1)$ & $\mathbb{Q}(-2)^{2}$ & 0 & $\mathbb{Q}(-3)$ \\
\hline
\end{tabular}

proof: This follows from a direct local calculation. Around any point of $Q=Z_{0} \cap P$ the divisor $Z_{0}+2 P$ is given by an equation of the form $x y^{2}=0$. The 2 -fold cover then has equation $x y^{2}+z^{2}=0$, which has a smooth normalisation. Clearly, the map $\widetilde{P} \rightarrow P$ is a two fold cover, ramified precisely along the conic bundle $Q$. In other words, the composition $\rho: \widetilde{P} \rightarrow P \rightarrow L$ represents this threefold as a quadric bundle, with 
four fibres with an isolated singular point over the points $\Sigma$. We can determine the cohomology of $\widetilde{P}$ using the Leray spectral sequence of the map $\rho: \widetilde{P} \rightarrow L$. We find

$$
R^{0} \rho_{*}\left(\mathbb{Q}_{\widetilde{P}}\right)=\mathbb{Q}_{L}, \quad R^{1} \rho_{*}\left(\mathbb{Q}_{\widetilde{P}}\right)=0=R^{3} \rho_{*}\left(\mathbb{Q}_{\widetilde{P}}\right), \quad R^{4} \rho_{*}\left(\mathbb{Q}_{\widetilde{P}}\right)=\mathbb{Q}(-2) .
$$

The sheaf $R^{2} \rho_{*}\left(\mathbb{Q}_{\widetilde{P}}\right)$ is more interesting. As $H^{2}$ of of a quadric is generated by its two rulings, which get interchanged upon surrounding a point of $\Sigma$, and coalesce over $\Sigma$, we have

$$
R^{2} \rho_{*}\left(\mathbb{Q}_{\widetilde{P}}\right)=\pi_{*} \mathbb{Q}_{E}(-1),
$$

where $\pi: E \longrightarrow L$ is the elliptic curve, two-fold covering $L$ and ramifying over $\Sigma$. The Leray-spectral sequence degenerates and we can read off directly the cohomology groups, as Hodge structures.

The monodromy weight spectral sequence converges to the cohomology of $H^{k}\left(\widetilde{Z}_{\infty}, \mathbb{Q}\right)=$ $H^{k}\left(Y_{\infty}, \mathbb{Q}\right)$ and is determined from the intersections of the irreducible components of the semi-stable fibre, see e.g. [20], [16]. In our case there are only two components and a single intersection, so the $E_{1}^{p, q}$-page is very simple and looks like

$$
\begin{aligned}
& H^{4}(Q)(-1) \quad \longrightarrow \quad H^{6}\left(Z_{0}\right) \oplus H^{6}(\widetilde{P}) \quad \longrightarrow \quad 0 \\
& H^{3}(Q)(-1) \quad \longrightarrow \quad H^{5}\left(Z_{0}\right) \oplus H^{5}(\widetilde{P}) \longrightarrow 0 \\
& H^{2}(Q)(-1) \quad \longrightarrow \quad H^{4}\left(Z_{0}\right) \oplus H^{4}(\widetilde{P}) \longrightarrow H^{4}(Q) \\
& H^{1}(Q)(-1) \quad \longrightarrow \quad H^{3}\left(Z_{0}\right) \oplus H^{3}(\widetilde{P}) \longrightarrow H^{3}(Q) \\
& H^{0}(Q)(-1) \longrightarrow H^{2}\left(Z_{0}\right) \oplus H^{2}(\widetilde{P}) \longrightarrow H^{2}(Q) \\
& 0 \longrightarrow H^{1}\left(Z_{0}\right) \oplus H^{1}(\widetilde{P}) \longrightarrow H^{1}(Q) \\
& 0 \longrightarrow H^{0}\left(Z_{0}\right) \oplus H^{0}(\widetilde{P}) \longrightarrow H^{0}(Q)
\end{aligned}
$$

As we have determined all groups appearing, the cohomology-diagram has the following form:

$\begin{array}{ccccc}\mathbb{Q}(-3) & \longrightarrow & \mathbb{Q}(-3) \oplus \mathbb{Q}(-3) & \longrightarrow & 0 \\ 0 & \longrightarrow & 0 \oplus 0 & \longrightarrow & 0 \\ \mathbb{Q}(-2)^{6} & \longrightarrow & \mathbb{Q}(-2)^{46} \oplus \mathbb{Q}(-2)^{2} & \longrightarrow & \mathbb{Q}(-2) \\ 0 & \longrightarrow & H^{3}\left(Z_{0}, \mathbb{Q}\right) \oplus H^{1}(E, \mathbb{Q})(-1) & \longrightarrow & 0 \\ \mathbb{Q}(-1) & \longrightarrow & \mathbb{Q}(-1)^{46} \oplus \mathbb{Q}(-1)^{2} & \longrightarrow & \mathbb{Q}(-1)^{6} \\ 0 & \longrightarrow & 0 \oplus 0 & \longrightarrow & 0 \\ 0 & \longrightarrow & \mathbb{Q} \oplus \mathbb{Q} & \longrightarrow & \mathbb{Q}\end{array}$

As we know that the cohomology groups $H^{k}\left(Y_{\infty}, \mathbb{Q}\right)$ of the limit are pure Hodge structures, the maps at the left are injective, those on the right surjective, and the cohomology of $H^{k}\left(Y_{\infty}, \mathbb{Q}\right)$ comes out the right way:

$$
H_{\lim }^{3}\left(Y_{0}, \mathbb{Q}\right)=H^{3}\left(Z_{0}, \mathbb{Q}\right) \oplus H^{1}(E, \mathbb{Q})(-1) .
$$

\section{OutLOOK}

The family $\mathcal{X} \longrightarrow \Delta$ extends naturally to a projective family over $\mathbb{P}^{1}$ with four singular fibers at $\{0,1,2, \infty\}$. The fibers at 1 and 2 are double octic arrangements No. 3 and No. 19 respectively, whereas at $\infty$ we get degenerate configuration

$$
u^{2}=x y(x+y) z v^{3}(y+z+v) .
$$

The map

$$
(x, y, z, v, u) \longmapsto\left(-x, x+y, z, \frac{1}{t-1} v, u\right)
$$


defines an isomorphism between $\mathcal{X}_{\frac{t}{t-1}}$ and the quadratic twist of $\mathcal{X}_{t}$ by $1-t$, consequently quadratic base-changes of the family $\mathcal{X}$ ramified at 1 and $\infty$ are isomorphic. There are 63 one-parameter families of double octics listed in [13], which lead to 63 families of Calabi-Yau threefolds with $h^{12}=1$. In these families there are five more examples with a similar behaviour. Below one list all six cases, which come as three pairs:

\begin{tabular}{|r|l|r|r|}
\hline No. & equation & $t_{0}$ & $\mathcal{X}_{t_{0}}$ \\
\hline \hline 153 & $\begin{array}{r}x y z t(x+y+z)(y+z+t) \times \\
\times(A x-B y+A t)(A x-B y+A z+A t)\end{array}$ & -2 \\
\hline 197 & $\begin{array}{l}x y z t(x-y-z+t)(A x+B y+B z) \times \\
\times(B y+B z+A t)(A x+B z+A t)\end{array}$ & $-\frac{1}{2}$ & 93 \\
\hline \hline 96 & $\begin{array}{l}x y z t(x+y)(x+y-z+t) \times \\
\times(A x-B y+B z+A t)(A y+B z+A t)\end{array}$ & -2 \\
\hline 100 & $\begin{array}{l}x y z t(x+y-z+t)(A x+A y+B z) \times \\
\times(A y+B z+A t)(B y-B z-A t)\end{array}$ & $-\frac{1}{2}$ \\
\hline \hline 155 & $\begin{array}{l}x y z t(A x+B y+A z)(A x+(A+B) y-B z+A t) \times \\
\times(A x-B z-B t)(A x+B y+A z+A t)\end{array}$ & $\frac{-1 \pm \sqrt{-3}}{2}$ \\
\hline 200 & $x y z t(x+y+z+t)(A x+A y-B z-B t) \times$ \\
& $\times(A y-B z+A t)(A x-B y-B t)$ & $\frac{-1 \pm \sqrt{-3}}{2}$ & $A$ \\
\hline
\end{tabular}

In the last column we have indicated the configuration number of the corresponding double octic from [13]. The symbol $A$ indicates a specific rigid Calabi-Yau manifold defined over $\mathbb{Q}(\sqrt{-3})$. The families No. 96 and No. 100 are in fact birational, as are No. 153 and No. 197. Families No. 155 and No. 200 have equal Hodge numbers and share the same Picard-Fuchs operators, but no birational map between them is known to us.

The degeneration of two fourfold points of type $p_{4}^{1}$ that collide and produce a $p_{5}^{1}$ that was analysed in this paper for No. 153 also occurs in No. 100 and No. 155. As a consequence we get again the central fiber singular along a double line with four pinch points. The only difference is that in the case of family No. 155 the $j$-invariant of four pinch-points equals 0 . The degenerations that occur in the other three cases No. 96, No. 197 and No. 200 are of a different kind: three double lines come together to form a triple line. This line is a double line of the singular element of the central fiber with four pinch point: one fivefold point and three fourfold points on this line. The $j$-invariant of this four points is again 1728 in first two cases and 0 in the last case.

The local exponents of the Picard-Fuchs operators in the first four families are all equal to $(0,1 / 2,3 / 2,2)$, which after quadratic base change become $(0,1,3,4)$, while in the case of the last two families No. 155, 200 they are $(0,1 / 2,5 / 2,3)$, which after a quadratic base change become $(0,1,5,6)$. It is surprising and beautiful to see the order of the automorphism group of the associated elliptic curve appear in the local exponents of the degeneration.

The first four families No. 96, 100, 153 and 197 are also birational to Kummer fibrations of rational elliptic surfaces. The degeneration of the corresponding fiber products results from the collisions of fibers

$$
\left(I_{2} \times I_{0}^{*}\right)+\left(I_{0} \times I_{0}\right) \longrightarrow\left(I_{2} \times I_{0}^{*}\right) \quad \text { or } \quad\left(I_{2} \times I_{0}^{*}\right)+\left(I_{2} \times I_{0}\right) \longrightarrow\left(I_{4} \times I_{0}^{*}\right)
$$


The singularities in the central fiber correspond to two copies of the singular fiber of type $I_{0}^{*}$. It may very well be possible to analyse the degeneration cohomologically from this description.

We believe that our degeneration also has an arithmetical version that may be of interest. Recently, in [12] and [3] a version Néron-Ogg-Shafarevich criterion for a family of K3 surfaces was formulated, which enables to detect good reduction of a K3 surface over the fraction field $K$ of a henselian local ring $R$ with residue field $k$ of characteristic $p>0$ by having the Galois representation

$$
G_{K} \rightarrow \operatorname{Aut}\left(H_{e t}^{2}\left(X, \mathbb{Q}_{\ell}\right)\right)
$$

unramified. Based on our example, we are inclined to think that no similar criterion can exist for Calabi-Yau threefolds. If we replace $t$ by a sufficiently large prime $p$ in the formula describing our double octic, and doing the corresponding modifications, we end up with with a Calabi-Yau variety $Y$ over the $p$-adic field $K=\mathbb{Q}_{p}$ for which we have the suspicion that the Galois representation

$$
G_{K} \rightarrow \operatorname{Aut}\left(H_{e t}^{3}\left(Y, \mathbb{Q}_{\ell}\right)\right)
$$

is unramified for $\ell \neq p$ and crystalline for $\ell=p$, but for which no good (terminal) reduction is in sight.

Acknowledgement: We thank Radu Laza for showing interest in this example. The first named author was partially supported by the National Science Center grant no. 2014/13/B/ST1/00133. This work was partially supported by the grant 346300 for IMPAN from the Simons Foundation and the matching 2015-2019 Polish MNiSW fund.

\section{REFERENCES}

[1] M. Atiyah, On analytic surfaces with double points, Proc. Roy. Soc. A 247(1958) 237 - 244.

[2] E. Brieskorn, Singular elements of semi - simple algebraic groups. Actes. Congrès Inter. Math. 2 (1970) $279-284$.

[3] B. Chiarellotto, C. Lazda, C. Liedtke, A Néron-Ogg-Shafarevich criterion for K3-surfaces, arXiv:1701.02945v4. [math. AG].

[4] S. Cynk, D. van Straten, Picard-Fuchs operators for octic arrangements I (The case of orphans), arXiv:1709.09752 [math.AG] (to appear in CNTP).

[5] S. Cynk, B. Kocel-Cynk, Classification of double octic Calabi-Yau threefolds, arXiv:1612.04364.(to appear in Commun. Contemp. Math.)

[6] W. Decker, G. M. Greuel, G.-Pfister, H. Schönemann, Singular 4-1-1 — A computer algebra system for polynomial computations. http://www.singular.uni-kl.de (2018).

[7] P. Deligne, Théorie de Hodge. I. Actes du Congrés International des Mathmaticiens (Nice, 1970), Tome 1, pp. 425-430. Gauthier-Villars, Paris, 1971.

[8] R. Friedman, A degenerating family of quintic hypersurfaces with trivial monodromy, Duke Math. J. 50 (1983), 203-214.

[9] R. Friedman, On Threefolds with Trivial Canonical Bundle, In:Complex Geometry and Lie Theory (eds. J. Carlson, H. Clemens, D. Morrison), Proc. Symp. Pure Math. 53, (1991), 103 - 134.

[10] J. Kollár, R. Laza, G. Sacca, C. Voisin, Remarks in degenerations of Hyper-Kähler Manifolds, arXiv:1704.02731 [math.AG].

[11] V. S. Kulikov, Degenerations of K3 surfaces and Enriques surfaces, Math. USSR Izvestija 11(1977), no. 5, $957-989$.

[12] C. Liedtke, Y. Matsumoto, Good reduction of K3 surfaces. Compos. Math. 154 (2018), no. 1, 135.

[13] C. Meyer, Modular Calabi-Yau threefolds. Fields Institute Monographs, 22, American Mathematical Society, Providence, RI, 2005.

[14] J. Morgan, Topological triviality of various analytic families, Duke Math, J. 50, no.1, (1983), 215-225. 
[15] U. Persson, H. Pinkham, Degeneration of Surfaces with Trivial Canonical Bundle, Ann. of Math. Vol. 113, No. 1 (1981), 45-66.

[16] C. Peters, J. Steenbrink, Mixed Hodge structures. Ergebnisse der Mathematik und ihrer Grenzgebiete. 3. Folge. A Series of Modern Surveys in Mathematics, 52. Springer-Verlag, Berlin, 2008.

[17] Groupes de monodromie en géométrie algébrique. I. (French) Séminaire de Géométrie Algébrique du Bois-Marie 19671969 (SGA 7 I). Dirigé par A. Grothendieck. Avec la collaboration de M. Raynaud et D. S. Rim. Lecture Notes in Mathematics, Vol. 288.

[18] Groupes de monodromie en géométrie algébrique. II. Séminaire de Géométrie Algébrique du BoisMarie 19671969 (SGA 7 II). Dirigé par P. Deligne et N. Katz. Lecture Notes in Mathematics, Vol. 340. Springer-Verlag, Berlin-New York, 1973.

[19] W. Schmid, Variation of Hodge structure: the singularities of the period mapping. Invent. Math. 22 (1973), 211-319.

[20] J. Steenbrink, Limits of Hodge structures. Invent. Math. 31 (1975/76), no. 3, 229-257.

[21] C. Voisin, Degenerations de Lefschetz et variations de structures de Hodge, J. Differential Geometry 31 (1990), 527-534. 